\title{
INTENSIFICACIÓN DEL PROCESO TECNOLÓGICO DE PRODUCCIÓN DE CAKE
}

\section{INTENSIFICATION OF THE TECHNOLOGICAL PROCESS OF CAKE PRODUCTION}

\author{
$\begin{array}{lll}\text { iD Julio Cesar Aragón Fontes } & & \text { (iD) Héctor Eduardo Sánchez Vargas } \\ & & \text { iD } \text { Amyrsa Salgado Rodríguez }^{3}\end{array}$ \\ 'Universidad de Camagüey "Ignacio Agramonte Loynaz", Cuba \\ Correspondencia: \\ Dr. Julio Cesar Aragón Fontes \\ julioaragonfontes@gmail.com
}

\section{RESUMEN}

Los retos que impone la producción de alimentos y las limitaciones tecnológicas y de aprovechamiento de capacidades que enfrentaba la dulcería objeto de estudio condujeron a establecer como objetivo de este trabajo intensificar el proceso de elaboración de Cake empleando técnicas y herramientas de la investigación de operaciones para el incremento productivo en la dulcería del Hotel Caonao. Como métodos y técnicas se aplicaron análisis-síntesis, revisión documental y herramientas propias de la investigación de operaciones. Se reconocieron como resultados, la determinación de la carga capacidad del proceso de producción durante la elaboración de Cake; diagrama de Gantt para el análisis de tres escenarios actual, con adecuaciones organizativas, con adecuaciones organizativas y modificaciones que incluye un proceso inversionista; una propuesta de modificaciones tecnológicas del sistema de trabajo en un turno de trabajo; el análisis de factibilidad de la inversión propuesta; y medidas organizativas en cuanto a cantidad de operarios y sus roles. Se concluyó que, el empleo del diagrama de Gantt puede ser muy efectivo para la mejora de los procesos de producción seriada; para incrementar los niveles productivos deberá accionarse en torno las operaciones de horneado y sobre la capacidad del equipamiento para producir merengue; las medidas técnico-organizativas, basadas en el solapamiento de las operaciones y ciclos productivos, así como en el proceso inversionista propuesto, pudieran incrementar los niveles productivos del Cake de 1 $800 \mathrm{~g}$ en 2,33 veces, manteniendo el mismo régimen productivo de 8 horas diarias y contratando a un operario, adicional a los ya existentes.

Palabras clave: análisis de procesos, producción seriada, productividad

\section{ABSTRACT}

The challenges imposed by food production and the technological limitations and the use of capacities faced by the confectionery under study led to the following objective of this work intensify the process of making Cake using techniques and tools from operations research to the increase in production at the Hotel Caonao candy store. As methods and techniques were applied: analysis-synthesis, documentary review and tools of operations research. The following were recognized as results the determination of the load capacity of the production process during the elaboration 
of Cake; Gantt chart for the analysis of three scenarios: current, with organizational adjustments, with organizational adjustments and modifications that, includes an investment process; a proposal for technological modifications of the work system in a work shift; the feasibility analysis of the proposed investment; and organizational measures in terms of number of operators and their roles. It was concluded that the use of the Gantt chart can be very effective for the improvement of serial production processes; To increase the production levels, the baking operations and the capacity of the equipment to produce meringue should be driven; The technical-organizational measures, based on the overlapping of operations and production cycles, as well as the proposed investment process, could increase the production levels of the $1800 \mathrm{~g}$ Cake by 2.33 times, maintaining the same 8-hour production regime daily and hiring an operator, additional to the existing ones.

Key words: process analysis, serial production, productivity

\section{INTRODUCCIÓN}

La garantía de una alimentación sana y sostenible se ha convertido en una preocupación a escala mundial. La Agenda 2030 para el desarrollo sostenible (CEPAL, 2018), hoja de ruta que promueve la CEPAL como oportunidad para el desarrollo sostenible de los países de América Latina y el Caribe, en su objetivo de desarrollo sostenible 2, apunta entre otros, la necesidad de asegurar la sostenibilidad de los sistemas de producción de alimentos.

Una de las tendencias a escala mundial que han sufrido los alimentos en los últimos años es el incremento de los precios de estos productos, en este sentido la región de América ha experimentado un incremento de alrededor de un ocho por ciento entre los años 2017 y 2020 (FAO, 2020).

El incremento de los precios constituye un desafío al que se enfrentan los decisores para trazar políticas dirigidas a la mejora de la eficacia y eficiencia de los procesos. Ello acompañado del atraso tecnológico, la baja disponibilidad de las materias primas y los recursos energéticos, el acceso a la información y la discreta preparación de los profesionales, comparada con la del mundo desarrollado. El impacto de las estructuras productivas y comerciales sobre la oferta y demanda de los alimentos constituye otro elemento desafiante (CELAC, 2020).

En este contexto juega un papel relevante, la capacidad de los decisores en el uso de métodos científicos para la solución de problemas y la toma de decisiones técnicas y administrativas que posibiliten mejoras sustanciales en la gestión de los procesos asociados a las actividad de producción de alimentos, con desarrollo sostenible desde los puntos de vistas técnico, económico y ambiental, de allí el papel de la Investigación de Operaciones como disciplina de carácter científico dedicada esencialmente al análisis y solución de problemas de organización, administración, planificación y control de sistemas, tanto en la naturaleza como en la sociedad (EAP, 2015). Esta aporta valiosas técnicas y herramientas para analizar a profundidad los procesos y arribar a soluciones efectivas, lo que supone, su aplicación impostergable y sistemática, esencialmente en los sectores relacionados con la producción de alimentos.

Dentro de la producción de alimentos se encuentra la pastelería. Esta constituye una actividad fundamental que tomó auge a finales del siglo XVIII, se incrementó con el consumo de postres y se perfeccionó con tipos de decoraciones, lo que propició una mayor aceptación, convirtiéndose en una de las grandes industrias de la alimentación a nivel mundial (Saavedra, 2006). Con el advenimiento de los medios mecánicos y automáticos de producción se ha hecho necesaria la estandarización de los productos y procesos, por lo que la elaboración debe realizarse de un modo científico, de manera que se garantice calidad uniforme y un mínimo de defectos.

En la actualidad, las políticas gubernamentales han forzado de alguna manera la revisión de los 
sistemas de gestión, en un entorno en el que la competencia mercantil exige a las empresas mejoras de la eficiencia y la productividad, de manera que ocurra una reducción de los costos de producción y la ampliación del mercado.

La empresa de alojamientos Camagüey es una empresa cubana de esta región del país, constituida en 1997, y que ha trazado una estrategia de perfeccionamiento empresarial. Dentro de esta entidad, la dulcería del Hotel Caonao tiene la peculiaridad de abastecer de productos de repostería a las distintas unidades empresariales a otros clientes de la provincia, pero su estructura productiva y obsolescencia tecnológica limita el incremento de los niveles productivos y las utilidades.

En la dulcería del Hotel Caonao se produce una amplia variedad de productos de pastelería, bizcochería, pasta choux, masas quebradas y otros de repostería fina, sin embargo, por su aceptación y posicionamiento en el mercado, se destaca la producción del llamado Cake, producto de batido de espuma muy conocido internacionalmente y de amplia tradición en el país.

Este producto se fabrica desde hace más de 60 años y se han mantenido por décadas altos estándares de calidad. Además de la aceptación de este producto, existe alta experticia en los trabajadores que intervienen en su proceso productivo y tienen la ventaja de su relativa facilidad de producción y la existencia de un número importante de presentaciones de este producto.

Se ha identificado al Cake como producto líder de la entidad y se quiere potenciar su producción ante un mercado promisorio, pero no se han realizado con anterioridad estudios que permitan identificar qué operaciones o equipos pudieran constituir elementos críticos del proceso, que de alguna manera limiten el incremento de los niveles productivos; por lo que no se conoce sobre qué parte del proceso actuar, ni qué inversiones realizar para elevar la productividad y las utilidades.

Para resolver la anterior problemática se requiere de la aplicación de herramientas de diagnóstico; así como, de análisis y diseño de procesos que, teniendo en cuenta las condiciones técnico organizativas actuales, permitan la concepción de un proceso técnico y económicamente factible de mayor productividad. Ante esta situación, se propone como objetivo de este trabajo, mejoras técnico-organizativas que contribuyan el incremento productivo en la dulcería del Hotel Caonao. Para cumplirlo se realizó un estudio de carga capacidad que permitió la identificación de la operación limitante del mismo, luego se propusieron y analizaron alternativas tecnológicas hasta realizar una propuesta de inversión evaluada a partir de la determinación y análisis de los indicadores dinámicos de factibilidad de la inversión, valor actual neto (VAN), tasa interna de retorno (TIR) y el plazo de recuperación de la inversión (PRI).

\section{MATERIALES Y MÉTODOS \\ Caracterización del proceso de producción actual de la dulcería del Hotel Caonao}

El proceso de producción actual de la dulcería del Hotel Caonao se caracterizó a partir de un análisis del proceso tecnológico y de la aplicación de un estudio da carga capacidad, tomando como referencia la producción de Cake, en su presentación rectangular mediano de $1800 \mathrm{~g}$, uno de los productos líderes de la empresa. En la Figura 1, se muestra el esquema del proceso de producción de cake, cuyas operaciones están estandarizadas en la documentación tecnológica del establecimiento.

Este proceso, como es característico en los de su tipo para la producción pastelera, es una producción seriada, que se caracteriza por una nomenclatura de artículos elaborados periódicamente por lotes que se repite de forma secuencial (García \& Sierra, 2013; Herrera, 2011; Kalenatic et al., 2009).

En el proceso tecnológico bajo estudio, la batición se realiza por el método tradicional, en el que se baten el huevo, el azúcar y la sal hasta alcanzar el volumen deseado; el agua puede añadirse a intervalos durante el proceso o completamente al final, y por último, la harina y el polvo de hornear. En el moldeo se utilizan bandejas de $65 \times 45 \mathrm{~cm}$, previamente engrasadas y empapeladas. La cocción se realiza en un horno 
eléctrico a $2100 \stackrel{\circ}{ } \mathrm{C}$, por un espacio de tiempo de 25 minutos.

Después de refrescada la panetela es desmoldeada, se le añade almíbar y se viste y se decora con colores a gusto del consumidor.

Para la caracterización del proceso se incluyó un estudio de tiempo del tipo cronometraje, que es la técnica utilizada con mayor frecuencia (Rico et al., 2005). Este estudio permitió determinar el tiempo de cada una de las operaciones del proceso, la identificación de los equipos y sistemas involucrados y la definición del criterio de lote, lo que permitió definir la cantidad de productos que se producen en un lote o ciclo productivo y posteriormente, teniendo en cuenta el régimen productivo y el fondo de tiempo disponible anual, se pudo determinar la capacidad productiva anual.

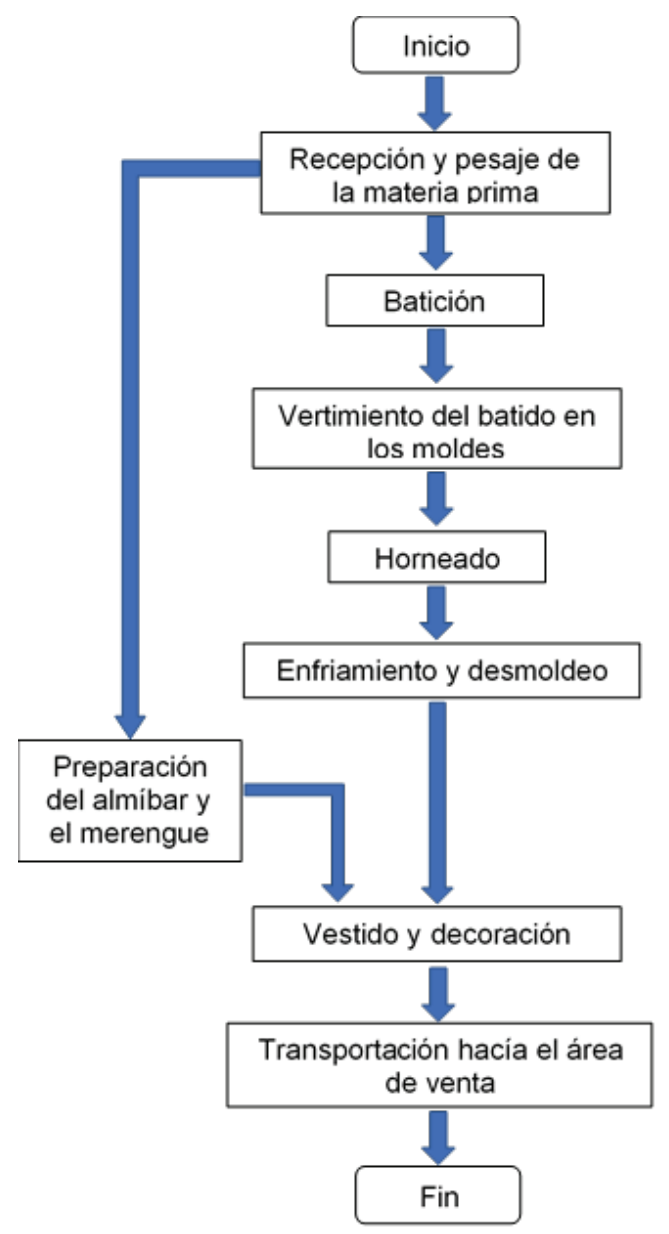

Figura 1. Esquema tecnológico del proceso de producción de cake

Posterior al estudio de tiempo y con el resto de la información recopilada, se confeccionó un diagrama Gantt del proceso para un turno de trabajo de 8 horas, que representó secuencialmente al proceso productivo. Los gráficos o diagramas de Gantt fueron concebidos por el ingeniero norteamericano Henry L. Gantt, uno de los precursores de la ingeniería industrial contemporánea y forma parte de las herramientas de la investigación de operaciones.
A través de ella, se realiza la programación de actividades, es decir, su distribución conforme a un calendario, de manera tal que, se pueda visualizar el periodo de duración de cada actividad y el tiempo total requerido para la ejecución de un proceso o proyecto. Desde su creación, esta ha sido un instrumento muy adaptable y de uso universal, dado por su facilidad de construcción y utilidad para identificar la actividad en que se estará utilizando cada uno de los recursos, de 
modo que, puedan evitarse periodos ociosos innecesarios (Moreno \& Gutiérrez, 2007). Por todas estas razones, es una herramienta universal empleada por autores como Beltrán et al. (2009), Bravo (2009) y Cortés \& González (2013), en los estudios de mejora de procesos y en actividades de planificación.

La aplicación de Gantt permitió determinar el tiempo total que se invierte en la realización de un ciclo productivo, el número de ciclos productivos diarios e identificar la operación limitante del proceso. Con estos elementos se determinó la capacidad productiva diaria y anual. Su análisis también facilitó la identificación de posibles modificaciones organizativas al proceso dirigidas al incremento de la capacidad productiva. La determinación de la operación limitante permitió dirigir el análisis hacia las modificaciones tecnológicas que permitirían un incremento en la productividad del proceso, que se deben realizar sobre esa misma operación limitante. La operación, etapa, punto limitante o cuello de botella, como también se le llama, es la actividad de menor capacidad total en el proceso y por lo tanto lo limita (Marsán et al., 2011). Los tiempos de duración de cada una de las operaciones constituyen un factor primordial para el diseño y análisis de procesos discontinuos (Albernas et al., 2012) y solo actuando sobre la operación limitante es posible lograr incrementos de capacidad en procesos secuenciales o consecutivos.

Estos elementos, condujeron a que se realizaran propuestas de modificaciones tecnológicas y se establecieran sistemas de trabajo sobre la base del incremento continuo de las capacidades productivas y el logro de niveles superiores de ganancias. Estas modificaciones se resumen con el establecimiento de los siguientes sistemas de trabajo:

a) Sistema de trabajo actual.

b) Con adecuaciones organizativas.

c) Con adecuaciones organizativas y modificaciones en la operación limitante que incluyen un proceso inversionista.

Por último, se realizó un análisis comparativo de los anteriores sistemas de trabajo desde el punto de vista de la capacidad productiva.

Además, se llevó a cabo un análisis de factibilidad de la inversión, empleando los conocidos indicadores de factibilidad VAN, TIR y PRI. También se propuso la cantidad de operarios y sus roles en el proceso productivo.

\section{RESULTADOS}

Como información previa a la determinación de la capacidad del proceso se constató que, en las condiciones técnico organizativas existentes en la dulcería del Hotel Caonao, se labora con un régimen de trabajo, un turno diario de 8 horas y una productividad que permite producir 105 cake diarios con tres ciclos productivos de 35 cake cada uno y la labor de cuatro operarios.

El estudio de tiempo realizado arrojó los resultados que se muestran en la Tabla 1 . En esta se relacionan las operaciones del proceso con los respetivos tiempos operativos relativos a la producción de un lote o ciclo productivo.

Tabla 1

Resultados del estudio de tiempo en el proceso de producción de cake

\begin{tabular}{llc}
\hline $\mathrm{N}^{\mathrm{o}}$ & \multicolumn{1}{c}{ Operaciones } & Tiempo (min) \\
\hline 1 & Recepción y pesaje de la materia prima & 15 \\
2 & Batición & 30 \\
3 & Vertimiento del batido en los moldes & 10 \\
4 & Horneado & $70^{*}$ \\
5 & Enfriamiento & $30^{*}$ \\
6 & Desmoldeo & $30^{*}$ \\
7 & Preparación del almíbar y el merengue & 40 \\
8 & Vestido y decoración & 45 \\
\hline
\end{tabular}


Las operaciones de horneado, enfriamiento y desmoldeo (marcadas con ${ }^{*}$ ) tienen necesariamente que realizarse en dos etapas, ya que la capacidad del horno no permite realizarlas en una sola etapa.

Además de la determinación del tiempo de las operaciones correspondientes a un ciclo productivo, también se determinaron los tiempos inherentes a la jornada laboral de 8 horas que se relacionan a continuación:

- Tiempo de preparación de la producción al inicio de la jornada laboral - 25 minutos

- Tiempo para la limpieza y la organización al finalizar la jornada laboral - 40 minutos.

- Tiempo para la transportación hacía el área de venta - 50 minutos.

En el tiempo de preparación, se realiza la extracción de las materias primas del almacén, se revisa y prepara el equipamiento, se acopia el agua necesaria para el proceso y se prepara el personal con los medios de protección, entre otras actividades.

La transportación de los productos terminados hacia el área de venta puede realizarse durante el periodo de tiempo correspondiente al ciclo productivo en el que se produce o puede realizarse al finalizar el turno de trabajo. En la actualidad, teniendo en cuenta que existe el espacio suficiente para almacenar y conservar los productos en el área de fabricación, esta operación se realiza al finalizar el turno de trabajo, momento en que existe mayor disponibilidad del personal para ejecutarla.

\section{Incremento productivo por acciones organizativas}

A partir de los resultados de este estudio de tiempo se confeccionó un diagrama Gantt del proceso productivo de Cake (Figura 2). El diagrama se construyó, no solo teniendo en cuenta los resultados del estudio de tiempo, sino previendo la posibilidad del solapamiento de operaciones del proceso y de ciclos productivos. En la Figura 2 (a), se representan los dos primeros ciclos del proceso y se incluye el periodo dedicado a la preparación de la jornada laboral. En la Figura 2 (b), representa los dos últimos ciclos para un turno de trabajo de 8 horas, incluyéndose el periodo dedicado a la limpieza y organización; así como, la transportación de los productos hacía el área de venta, de colores azul y carmelita respectivamente. La representación del ciclo intermedio carece de importancia al ser idéntico a los dos primeros ciclos.

Se puede apreciar que con el solapamiento de las operaciones de un mismo ciclo productivo se reduce el tiempo del ciclo total de éste, cuyo efecto, combinado con el solapamiento entre un ciclo productivo y otro, incrementa el número de ciclos que se pueden desarrollar en un turno o jornada laboral, lo que trae consigo un aumento de la productividad respecto a los procesos en los que no se realiza el solapamiento.

A pesar de, que la suma de los tiempos de todas las operaciones del proceso es de 270 minutos, según se muestra en la Tabla 1 , la posibilidad de solapamiento de las operaciones posibilita la reducción del tiempo del ciclo hasta 200 minutos. Como se puede apreciar en el diagrama, en este caso se han solapado las operaciones de horneado, enfriamiento, desmoldeo y preparación de almíbar y merengue. Para las operaciones de enfriamiento y desmoldeo este solapamiento ha sido posible a partir de la terminación de la primera etapa del horneado. La operación de preparación de almíbar y merengue se puede solapar, ya que, es una operación independiente, tal como, se ha representado en la Figura 1, y no requiere de la terminación de ninguna otra operación. 


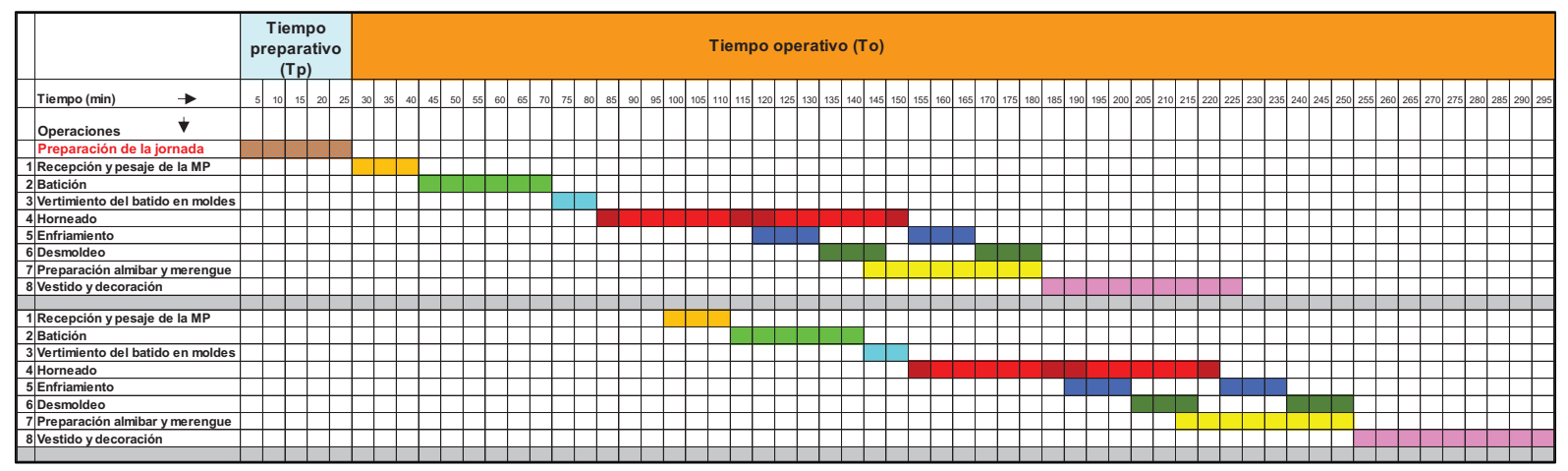

2 (a)

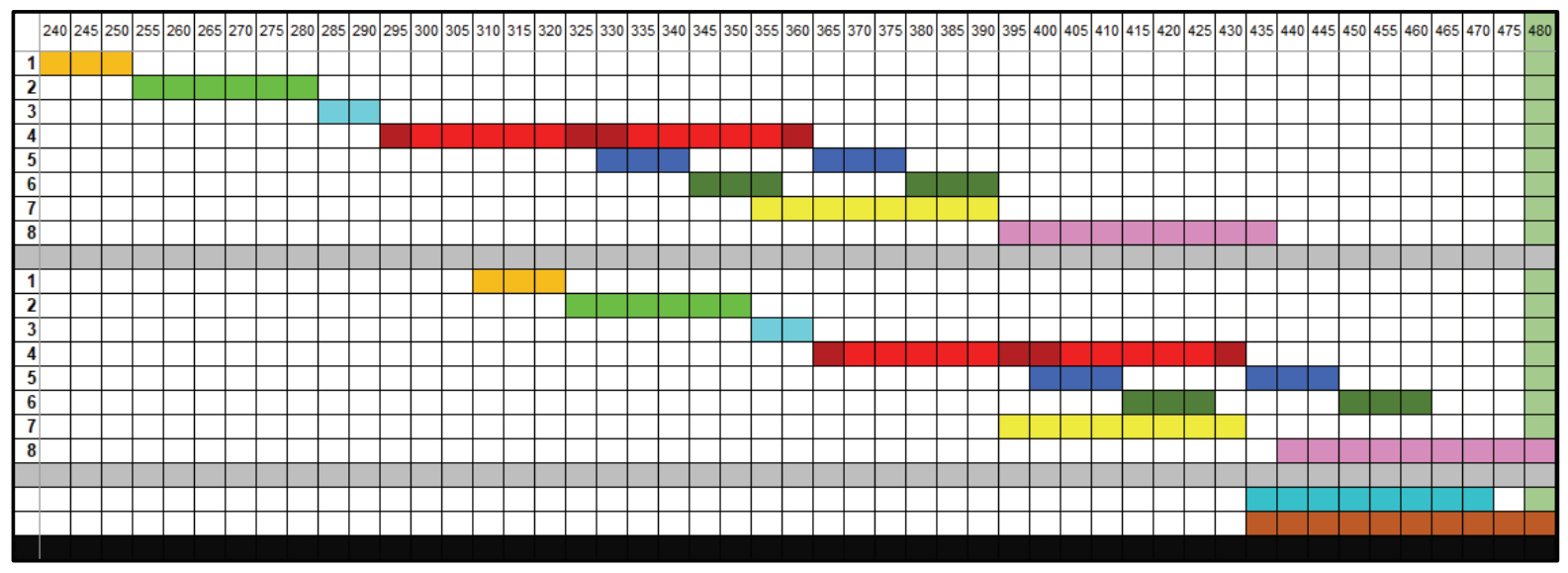

2 (b)

Figura 2. Diagrama Gantt del proceso después de acciones organizativas. 2 (a) Dos primeros ciclos productivos. 2 (b) Dos últimos ciclos productivos.

Como se evidencia, tanto en la Tabla 1 como en la Figura 2, la operación más extensa es el horneado con una duración de 70 minutos, de allí que constituye la operación limitante del proceso.

En este tipo de procesos de producción por lotes, cuando existen limitaciones de equipamiento y personal no se pueden llevar a cabo operaciones en paralelo y se necesita terminar una operación para comenzar la otra; sin embargo, la posibilidad de solapar operaciones de diferentes ciclos productivos conduce a un esquema productivo en el que la operación limitante determina la capacidad del proceso. Se puede observar en el esquema (Figura 2), que después de realizar los solapamientos entre operaciones y ciclos, la diferencia de tiempo entre dos operaciones análogas de ciclos diferentes es igual al tiempo de la duración de la operación limitante (70 minutos).

Con este análisis se infiere que, si se pretende incrementar la productividad del proceso, se deben desarrollar estrategias que reduzcan el tiempo de duración de la operación limitante. Estas estrategias deben estar dirigidas a: la utilización de unidades en paralelo, la combinación y descomposición de tareas, la utilización de almacenamiento intermedio y la aplicación de reglas de transferencia entre tareas (Albernas et al., 2012).

Con el establecimiento de este sistema de trabajo se garantiza que las operaciones que utilizan las mismas máquinas o son desarrolladas por un único personal no pueden coincidir en tiempo. Por esta razón, las operaciones análogas de diferentes ciclos cumplen esta condición. También deben cumplirla las operaciones de batición y preparación del almíbar y el merengue, que se llevan a cabo con el mismo equipamiento. En el caso de, la operación de vestido y decoración, que es netamente manual, el solapamiento no puede realizarse porque es ejecutada por un personal cuya carga no permite 
dicho solapamiento.

En el diseño del nuevo proceso se ha podido aprovechar el hecho ya explicado de la independencia de la operación de preparación de almíbar y merengue. En el último ciclo del turno de trabajo se podrá adelantar esa operación ante la carencia de un próximo ciclo en el que se desarrolle nuevamente una operación de batición que se desarrolla en con el mismo equipamiento. Por esta razón, la ejecución de la operación de preparación de almíbar y merengue puede comenzar inmediatamente después de terminada la misma operación del ciclo anterior. Esta estrategia reduce el tiempo del último ciclo en 25 minutos, por lo que tendría entonces solo 175 minutos, y se logra concebir un sistema de trabajo que en el que se podrían desarrollar cinco ciclos productivos en un turno de trabajo de 8 horas en lugar de los tres actuales.

\section{Incremento productivo por procesos inversionistas}

Retomando el posible uso de estrategias sobre la operación limitante (operación de horneado) para incrementar la productividad, se evaluó la utilización de unidades en paralelo, a partir del empleo de un segundo horno de igual capacidad que el instalado actualmente. Se identificó que esta es la operación limitante debido a limitaciones en la capacidad del horno actual. Igualmente, se identificó que la batidora constituye otro equipo limitante y la suma de las operaciones de batición y preparación de almíbar y merengue, que se realizan empleando este único equipo, también tienen una duración de 70 minutos, al igual que el horneado actual. De esta manera, se llegó a la conclusión de que, para lograr un incremento de la productividad en el proceso habría que actuar sobre un aumento de la capacidad del horno y de la batidora.

En la Figura 3, se muestra el diagrama Gantt que correspondería al proceso después de llevado a cabo un proceso inversionista con la compra de un horno de igual capacidad que el usado actualmente y para ser empleado en paralelo con el actual. También, la compra de una batidora que se emplearía en paralelo con la utilizada en las operaciones actuales de batición y preparación de almíbar y merengue. En el propio diagrama se observa que estas dos operaciones no coincidirán en tiempo, en el nuevo esquema tecnológico al existir la posibilidad de usar dos equipos en paralelo.

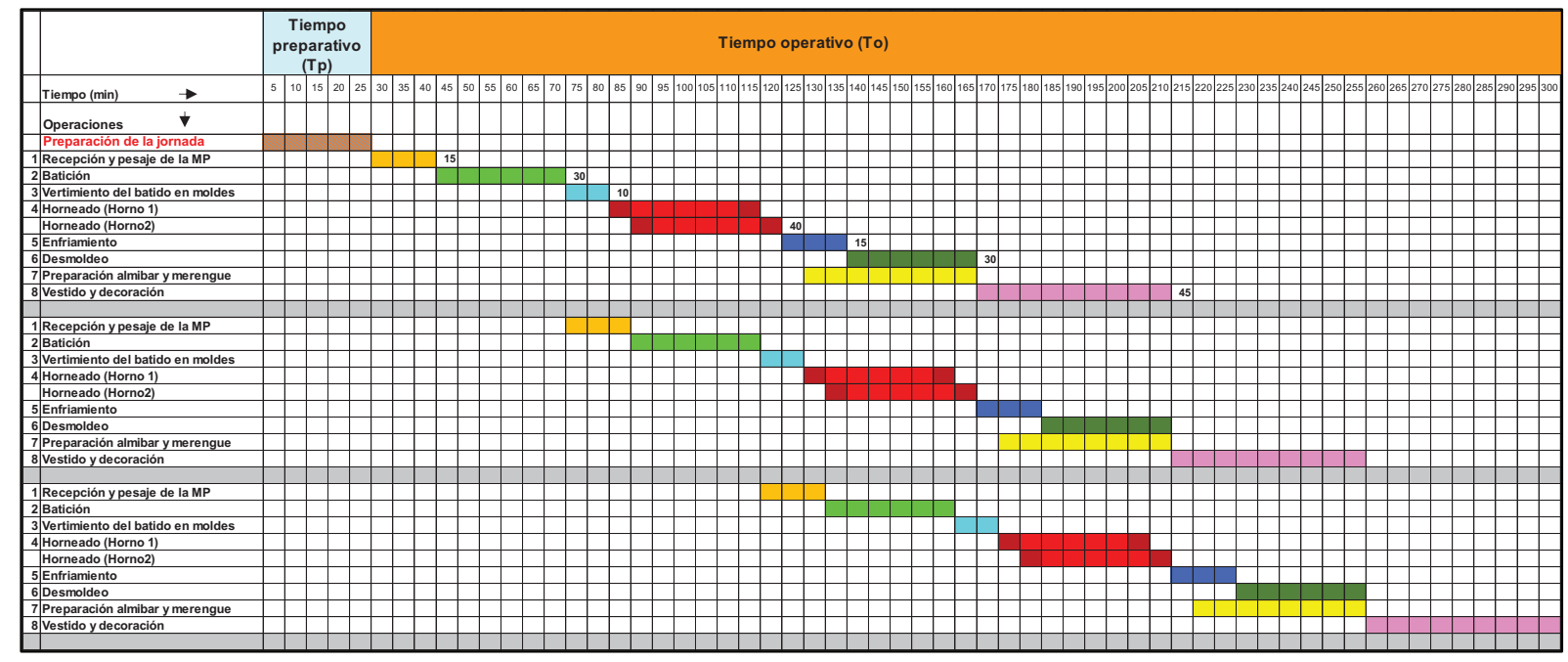

Figura 3. Diagrama Gantt del proceso después del proceso inversionista. Para los tres primeros ciclos productivos.

En el diagrama, al final de la barra que corresponde a cada operación aparece un número que indica el tiempo de duración de la operación en minutos. Se puede observar que, después de realizada la inversión, la nueva operación limitante es la operación vestido y decoración, con una duración de 45 minutos. En futuros estudios dirigidos al incremento de la capacidad del proceso, no se puede ignorar el estudio de esta operación. 
Con este nuevo sistema de trabajo, alcanzado luego de las referidas inversiones, se tendría un ciclo con un tiempo total de 185 minutos. Con el correspondiente solapamiento de ciclos productivos, el número de estos pudiera llegar a siete en una jornada laboral de 8 horas fabricarían 245 cake en ese periodo de tiempo. Este volumen de producción se corresponde con una cantidad 2,33 veces superior a la actual.
Para implementar este último sistema productivo, además de la compra e instalación del nuevo equipamiento, sería necesario además, la contratación de un operario que se le sumaría a los cuatro existentes, llegándose a la cantidad de cinco operarios, que deberían jugar los roles asignados, según su carga de trabajo que se muestran en la Tabla 2.

Tabla 2

Roles asignados a los operarios en el proceso productivo

\begin{tabular}{cl}
\hline $\begin{array}{c}\text { Cantidad de } \\
\text { operarios }\end{array}$ & \multicolumn{1}{c}{ Operaciones } \\
\hline 2 & $\begin{array}{l}\text { Recepción y pesaje de la materia prima } \\
\text { Vestido y decoración } \\
\text { Batición } \\
\text { Vertimiento del batido en los moldes } \\
\text { Horneado } \\
\text { Enfriamiento } \\
\text { Desmoldeo } \\
1\end{array}$ \\
& Preparación del almíbar y el merengue \\
\hline
\end{tabular}

Los operarios asociados a las operaciones de horneado y preparación de almíbar y merengue pudieran dedicarse a las operaciones de limpieza y organización después de concluido su último ciclo operacional. El operario asociado a la operación de batición pudiera dedicarse a la transportación del producto hacía el área de venta después de concluido su último ciclo operacional. Para desarrollar estas actividades se contaría con tiempo suficiente en el último periodo del turno de trabajo.

\section{Análisis de factibilidad de la inversión}

La ficha de costo del producto fue ajustada luego de los cambios tecnológicos propuestos, determinándose que el costo unitario sería igual a 67,85 CUP/cake. El costo anual total se determinó teniendo en cuenta la producción de 245 unidades diarias de cake, según la capacidad determinada después de implementados los cambios organizativos y tecnológicos. También se tuvo en cuenta que se laborará durante 8 horas diarias durante 305 días al año. Derivado de este análisis se determinó que la producción anual sería de 74725 unidades y el costo de producción anual de 5070 091,25 CUP/año.
El costo de inversión, incluye el costo de adquisición del equipamiento tecnológico; así como, los asociados a la transportación y montaje. La adquisición se refiere a un horno de igual capacidad y características del horno actualmente instalado en la dulcería y a una batidora planetarias similar a la existente. Como precios de referencias se utilizaron los tomados de los sitios web, https://spanish. alibaba.com para el precio del horno y https:// www.solostocks.com para la batidora Horno - 7 500,00 USD y Batidora - 2 396,68 EUR. Según la tasa de cambio vigente de conversión del dólar estadounidense (USD) y el euro (EUR), los costos de adquisición en CUP serían, Horno 180 000,00 CUP y Batidora - 64 422,80 CUP.

La suma de los costos de adquisición de estos dos equipos asciende a la suma de 244 422,75 CUP. Teniendo en cuenta los gastos de transportación e instalación, el monto total de la inversión asciende a 260 422,75 CUP/año.

Con los 74725 cake que se deben producir en el año, las ventas serían de 11956000 CUP. 
Partiendo de que el año cero es considerado como el año de la inversión y que en el primer año solo se produciría el $80 \%$ de la capacidad de la línea productiva, se tienen los resultados de los indicadores económicos financieros que se muestran en la Tabla 3, correspondiente a tres años después del año de la inversión.

\section{Tabla 3}

\section{Indicadores económicos asociados a la inversión}

\begin{tabular}{rrrrr}
\hline Año & Inversión inicial & Ventas anuales & $\begin{array}{c}\text { Costo de } \\
\text { Producción }\end{array}$ & $\begin{array}{c}\text { Flujo de caja } \\
\text { neto }\end{array}$ \\
\hline 0 & 260422,75 & 0,00 & 0,00 & $-260422,75$ \\
1 & & 9564800,00 & 4056073,93 & 5508727,00 \\
2 & 11956000,00 & 5070091,25 & 6885908,75 \\
3 & 11956000,00 & 5070091,25 & 6885908,75 \\
\hline
\end{tabular}

Con los flujos de caja obtenidos, mostrados en la Tabla anterior y usando una tasa de descuento anual del $10 \%$, se determinaron el valor actual neto (VAN) y la tasa interna de retorno (TIR), indicadores de la factibilidad de la inversión que se muestran en la Tabla 4. Esto se realizó utilizando las funciones de Excel NPV e IRR.

\section{Tabla 4 \\ Valor actual neto y tasa interna de retorno asociados a la inversión}

\begin{tabular}{ccrl}
\hline Año & $\begin{array}{c}\text { Tasa de descuento } \\
\text { anual }\end{array}$ & VAN & TIR \\
\hline 0 & $10 \%$ & 15611829,73 & $2138.69 \%$ \\
1 & $20 \%$ & 13096965,06 & \\
2 & $30 \%$ & 11185794,25 & \\
3 & $40 \%$ & 9697042,96 & \\
\hline
\end{tabular}

Los elevados valores positivos del VAN y la TIR, indican que el proyecto de inversión es altamente viable y la recuperación de la inversión se lograría en menos de dos meses.

Con las acciones técnico - organizativas propuestas en el presente trabajo se pudieran lograr incrementos significativos en los niveles productivos de la dulcería del Hotel Caonao. En un primer análisis, el estado actual se pudiera modificar solamente con acciones organizativas sobre la base del solapamiento de operaciones y ciclos productivos y algunas medidas dirigidas al aprovechamiento más eficaz de los recursos humanos y la definición de roles en el proceso productivo. En otro análisis, realizado a partir de la identificación de las operaciones y equipos limitantes, se determinó que, se pudiera alcanzar otro incremento muy significativo de la producción. Ambos análisis realizados tomando como base la producción del producto líder
Cake. Las Figuras 4(a), 4(b) y 4(c), muestran los resultados de estas acciones.

En la Figura 4, se muestra el importante incremento que pudieran tener los niveles productivos y de venta luego de la implementación de las medidas técnicoorganizativas, entre las que se encuentran las acciones de solapamiento de operaciones y ciclos productivos; así como, las inversiones que se proponen para las operaciones de horneado, batición y preparación de merengue. Para ilustrar ese incremento se definieron tres estadios: (1) el momento actual; (2) medidas organizativas y de solapamiento de operaciones y ciclos; (3) medidas organizativas y de solapamiento de operaciones y ciclos más la inversión propuesta. Las Figuras 4(a) y 4(b), se refieren al incremento de la producción diaria y anual y la Figura 4(c), indica el incremento de las ventas anuales. 


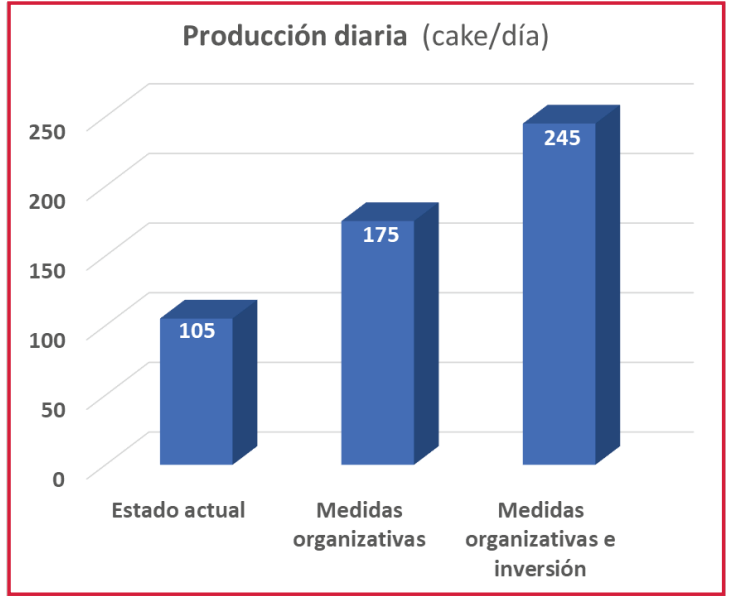

4 (a)

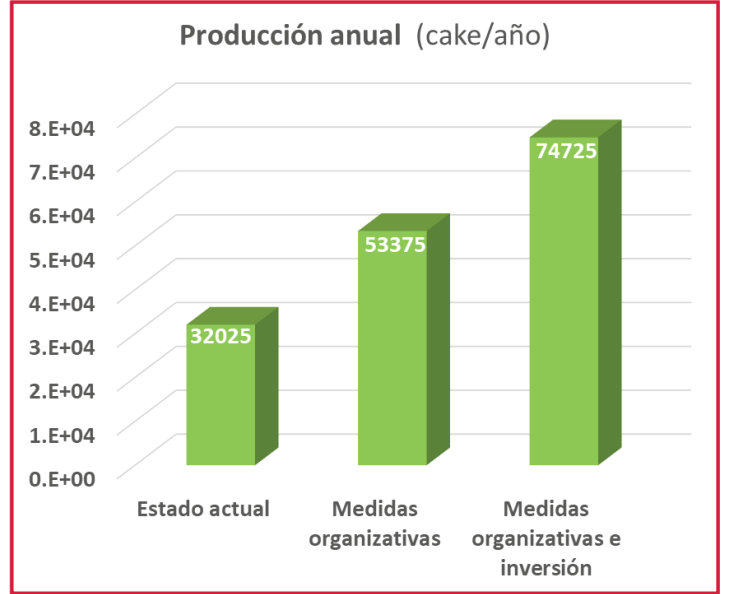

4 (b)

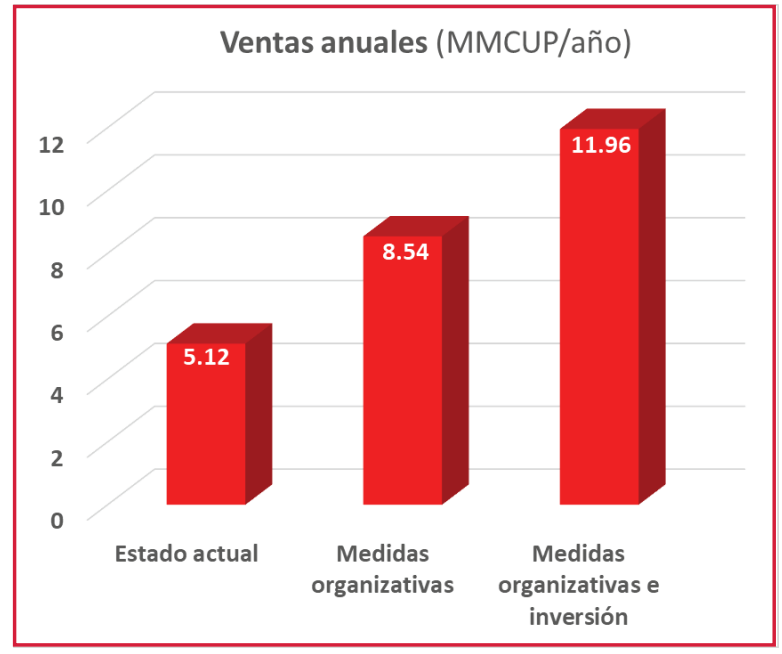

4 (c)

Figura 4. Resultados de la producción diaria 4 (a); Producción anual 4 (b); Ventas anuales 4 (c)

Los tres gráficos que aparecen en la Figura 4 , constituyen una muestra irrefutable del crecimiento que originan las medidas técnicosorganizativas en la producción diaria, anual y en las ventas anuales, a partir de la aplicación de las herramientas de análisis e intensificación de procesos que aporta la investigación de operaciones.

\section{CONCLUSIONES}

1. El empleo del diagrama de Gantt, como herramienta de organización y planificación, puede ser muy efectiva para la mejora de los procesos de producción seriada en la que los productos son elaborados por lotes que se repiten de forma secuencial.

2. Para incrementar los niveles productivos, en las condiciones actuales de la dulcería del Hotel Caonao, se debe considerar la realización de acciones en torno a las operaciones de horneado y sobre la capacidad del equipamiento asociado a la batición y producción de merengue. Estas tres operaciones están limitadas por la capacidad del equipamiento.

3. Las medidas técnico-organizativas, basadas en el solapamiento de las operaciones y ciclos productivos; así como, en el proceso inversionista propuesto, pudieran incrementar los niveles productivos del producto cake de $1800 \mathrm{~g}$ en 2,33 veces, manteniendo el mismo régimen productivo de 8 horas diarias y contratando solamente a un operario, adicional a los ya existentes. 


\section{REFERENCIAS BIBLIOGRÁFICAS}

Albernas, Y., González, M., González, E., Corsanol, G., \& Verelst, H. (2012). Procedimiento para la síntesis y diseño óptimo de plantas discontinuas (Parte I). Tecnología Química, XXXII(3), 257-264. Disponible en: http://scielo.sld.cu/scielo. php?script=sci_arttext \&pid=S2224$61852012000300005 \&$ lng=es\&tlng=es.

Beltrán, J., Carmona, M., Carrasco, R., Rivas, M., \& Tejedor, F. (2009). Guía para una gestión basada en proceso. Andalucía, España: Instituto Andaluz de Tecnología. Disponible en: https:// www.euskadi.eus/web01-s2ing/es/ contenidos/informacion/bibl_digital/ es_documen/adjuntos/Guia\%20para\%20 una\%20gestion-basada-procesos.pdf

Bravo, J. (2009). Gestión basada en proceso. Santiago de Chile, Chile: Editorial Evolución S.A. Disponible en: https://www.evolucion.cl/product-page/ gesti\%C3\%B3n-de-procesos-octavaedici\%C3\%B3n-1

CELAC. (2020). Seguridad Alimentaria bajo la Pandemia de COVID-19. DOI: https://doi.org/10.4060/ca8873es

CEPAL. (2018). La agenda 2030 y los objetivos de desarrollo sostenible. Una oportunidad para América Latina y el Caribe Santiago: Naciones Unidas. Disponible en: https://repositorio.cepal. org/bitstream/handle/11362/40155/24/ S1801141_es.pdf

Cortés, M., \& González, D. (2013). Sistema de gestión de la producción en la planta de panadería de la empresa cereales el líder S.C.A. [Tesis de pregrado]. Universidad Libre de Colombia, Bogotá, Colombia. Disponible en: https://repository.unilibre. edu.co/bitstream/handle/10901/9441/ Proy e c t o \% 20 d e \% $20 \mathrm{Gr}$ a d o. pdf? sequence $=1 \&$ is Allowed $=y$

EAP. (2015). Escuela Académico Profesional.Currículo2016-2021. Escuela de Investigación Operativa. Disponible en: https://viceacademico.unmsm.edu. pe/wp-content/uploads/2016/07/EAPde-Investigaci\%C3\%B3n-operativa.pdf

FAO. (2020). World Food and Agriculture - Statistical Pocketbook 2020. Disponible en: http://www.fao.org/3/cb1521en/ CB1521EN.pdf

García, A, \& Sierra, J. (2013). Optimización del proceso logístico interno de las marcas propias de supermercados líder en la línea de panadería y respostería [Tesis de pregrado]., Universidad Libre de Colombia, Bogotá, Colombia. Disponible en: https:// repository.unilibre.edu.co/bitstream/ handle/10901/9332/DOCUMENTO\%20 FINAL.pdf?sequence $=1$ \&isAllowed $=y$

Herrera, Y. (2011). Estudio de normación del proceso productivo de la UEB Producción y Empaque Doña Neli [Tesis de pregrado], Universidad Central "Marta Abreu" de las Villas, Santa Clara, Cuba. Disponible en: https://dspace.uclv.edu. cu/bitstream/handle/123456789/4025/ Yettel\%c2\%aOHerrera\%c2\%aOP\%c3\%a 9rez\%c2\%aO.pdf

Kalenatic, D., López, C., \& González, L. (2009). Modelo de ampliación de la capacidad productiva. Revista Ingeniería. 14(2), 67-77. DOI: https://doi. org/10.14483/23448393.2381

Marsán, J., Cuesta, A., García, C., \& Padilla, C. (2011). La organización del trabajo: Ingeniería de métodos (Tomo I). La Habana, Cuba: Ed. Félix Valera. Disponible en: https://fdiazca. files.wordpress.com/2020/06/ltmarzacc81n-organizaciocc81n-deltrabajo-tomo-1-ingeniericc81a-demecc81todos-2008.pdf

Moreno, L., \& Gutiérrez, A. (2007). Herramientas de lacalidadindispensables para la toma de decisiones [Tesis de 
pregrado], Centro Universitario Regional de Matagalpa - UNAN Managua, Managua,Nicaragua. Disponible en: https://repositorio.unan.edu.ni/7183/

Rico, L., Maldonado, A., Escobedo, M., \& de la Riva, J. (2005). Técnicas Utilizadas para el Estudio de Tiempos: un Análisis Comparativo. Instituto Tecnológico de Cd. Juárez. Cultura científica y tecnológica, 11, 1-10. Disponible en: http://erevistas. uacj. $\mathrm{mx} / \mathrm{ojs} /$ index.php/culcyt/article/ view/539

Saavedra, I. (2006). El panadero Dulcero. Ciudad de la Habana. Disponible en: https://isbn.cloud/9789591313294/ el-panadero-dulcero/ 